\title{
A little elaboration goes a long way: The role of generation in eyewitness suggestibility
}

\author{
SEAN M. LANE \\ Louisiana State University, Baton Rouge, Louisiana \\ AND \\ Maria S. Zaragoza \\ Kent State University, Kent, Ohio
}

\begin{abstract}
Although research has documented that perceptual elaboration (e.g., imagery) can increase false memory, prior research has not ascertained whether such effects are due to the act of generation or simply from exposure to perceptual details. Two experiments explored this question using the eyewitness suggestibility paradigm. Experiment 1 compared the effect of generating descriptions of suggested items with the effects of reading elaborated versions of the items or the suggested items alone. Experiment 2 compared participants who generated descriptions to participants who read the same descriptions. Generating a description increased false memory and increased accurate memory for the items' actual source, relative to comparable control conditions. Generation also increased claims of having a (false) vivid recollection of the items in the event. Overall, the results suggest that conditions that require people to describe the appearance of objects that they do not remember are even more pernicious than conditions that involve exposure to such details.
\end{abstract}

In real-world forensic interrogations, eyewitnesses are sometimes pressed to describe people, objects, or events that they do not remember well or never actually witnessed. For example, Dent (1982) described a situation in which a police officer interrogated a child who had witnessed an event involving three men and a woman. In the following quote, the officer inquired about the woman's poncho and cap, when in fact the woman in question did not wear anything on her head, nor did she wear a poncho:

Q: Wearing a poncho and a cap?

A: I think it was a cap.

Q: What sort of cap was it? Was it like a beret, or was it a peaked cap, or-?

A: No, it had sort of, it was flared with a little piece coming out. It was flared with a sort of button thing in the middle.

Q: What ... was it a peak like that, that sort of thing?

A: Ye-es ...

Q: That's the sort of cap I'm thinking you're meaning, with a little peak out there.

A: Yes, that's the top view, yes.

Q: Smashing, Um—what color.
A: $\mathrm{Oh}$ ! Oh-I think it was um, black or brown.

(pp. 290-291)

Although there were multiple suggestive influences present in this interrogation (e.g., reinforcement by the police officer and leading questions), this example appears to show that pressing the witness to describe the fictitious cap altered her memory for the witnessed event. Over the course of the interrogation, the witness, who was initially unsure whether she remembered a cap, appeared to gain confidence that she had actually seen the specific cap she had been pressed to describe.

Why might pressing witnesses to generate descriptions of suggested items promote the development of false memories? As is predicted by the source-monitoring framework (Johnson, Hashtroudi, \& Lindsay, 1993), evidence from a variety of domains has shown that memories of suggested events will be confusable for memories of "real" events to the extent that memories of suggested events contain features (e.g., sensory and perceptual details) that are similar to characteristics of memories of "real" events. For example, there is considerable evidence that visual imagery can serve as a potent catalyst to false memory creation (e.g., Dobson \& Markham, 1993; Garry, Manning, Loftus, \& Sherman, 1996; Goff \& Roediger, 1998; Gonsalves et al., 2004; Hyman \& Pentland, 1996; Lindsay, Hagen, Read, Wade, \& Garry, 2004), because imagining a suggested item imbues the memory representation of the suggested item with sensory and perceptual details similar to those of perceived

S. M. Lane, slane@Isu.edu 
events (see Drivdahl \& Zaragoza, 2001, and Thomas, Bulevich, \& Loftus, 2003, for more direct evidence for the role of sensory/perceptual elaboration in the development of false memories). As with imagination, one consequence of pressing witnesses to describe a poorly remembered or fictitious item is that it forces the witness to create a more specific, concrete, and perceptually detailed version of the suggested item. Hence, as in the interrogation illustrated above, pressing witnesses to describe suggested events may render the memory of the suggestion highly confusable for a memory from the witnessed event.

One issue that has not been addressed by previous research on imaginal elaboration and false memory is what role, if any, the act of generating a perceptually detailed representation might play in promoting false memory creation. ${ }^{1}$ If the amount of perceptual detail associated with a suggested memory is the primary predictor of false memory errors, it is possible that simply exposing participants to a perceptually detailed description of a suggested item or event (e.g., by having them read it) would lead to comparable increases in false memory. The existing literature cannot address this issue because the typical procedure is to compare performance in an imagination group (where participants are led to generate a perceptually elaborated version of the suggested event) with a comparison group that is not pressed to elaborate on the suggested information (whose resulting memory representation is likely to be lacking in sensory/perceptual detail). Because the generation of perceptual detail and the presence of perceptual detail have not been manipulated independently, the contribution of each variable to false memory development is difficult to assess.

The goal of the present study was to assess whether pressing eyewitnesses to generate descriptions of suggested (i.e., fictitious) items would increase false memory for these items, relative to simply reading descriptions of these items. Although there is a large body of empirical evidence on the impact of suggestive interviews on eyewitness testimony and memory, to our knowledge, no studies have directly assessed this issue. Indeed, because the bulk of the scientific literature on eyewitness suggestibility is based on an experimental paradigm where participants read the suggested information (e.g., either embedded in narrative descriptions of the event or presupposed in written questions about actually witnessed objects/events; although see, e.g., Hoffman, Loftus, Greenmun, \& Dashiell, 1992), the potential role of generation in eyewitness suggestibility is not well understood.

At this juncture, it is difficult to predict whether pressing participants to generate descriptions of suggested items might increase false memory, relative to reading descriptions of the same items. In such a situation, participants are called upon to produce plausible sensory/ perceptual and contextual details, presumably based on their memory for the actual event and/or semantic knowledge (e.g., of the item class). Extrapolating from research on the generation effect (e.g., Hirshman \& Bjork, 1988; Slamecka \& Graf, 1978), there are reasons to expect that, relative to reading, generating descriptions should improve memory for these descriptions and increase the extent to which these perceptual details are bound to their mental representation of the suggested event (see Burns, 1990, Hirshman \& Bjork, 1988, and McDaniel, Wadill, \& Einstein, 1988, for evidence that generation improves encoding of the characteristics shared by the cue and the target word, as well as the relationships between the items on the list). Hence, generating a detailed description of a suggested item may, like imagining a suggested item, result in a vivid, perceptually detailed representation that is easily confused for a memory based on perceptual experience. Furthermore, generating a description may lead participants to rely on idiosyncratic knowledge of the event or the item category. Such features, when accessed at test, may seem to be particularly compelling evidence that the suggested item was observed in the witnessed event because they are characteristic of the type of things a given person would typically notice about such an item (Zaragoza, Payment, Ackil, Drivdahl, \& Beck, 2001; see Stark, Perfect, \& Newstead, 2005, for similar arguments in the unconscious plagiarism paradigm).

On the other hand, because generation involves more effortful and controlled processing than does reading (and thus results in better memory for cognitive operations; see, e.g., Johnson \& Raye, 1981), participants who read descriptions of suggested items should be less likely to remember the actual source of the suggested items (i.e., that the suggestion was provided by a postevent source) than are participants who generate descriptions (see, e.g., Geghman \& Multhaup, 2004; Hicks, Marsh, \& Ritschel, 2002, Experiments 1 and 3; and Marsh \& Hicks, 1998, Experiment 1, for evidence that generation results in better source memory than does reading). ${ }^{2}$ Because poor source memory renders a memory trace more vulnerable to misattribution, it is possible that reading descriptions of suggested items might increase false memory, relative to generating them. We note, however, that in the eyewitness suggestibility paradigm (as in real-world forensic investigations), knowing that some piece of information came from a postevent interview does not necessarily imply that it was not witnessed (i.e., this information is not disqualifying; see Gallo, 2004) because much of the information encountered in the postevent session is true of the witnessed event. Hence, accurate memory for the suggested item's postevent source does not necessarily prevent the development of a false memory of having witnessed the suggested item (see Mitchell \& Zaragoza, 2001, for a discussion of this issue).

Finally, we note that pressing witnesses to generate descriptions of items that they do not remember witnessing differs in important ways from the types of generation tasks that have typically been studied in the literature (e.g., generating an antonym from a cue). Relative to reading, pressing witnesses to generate descriptions of suggested (i.e., fictitious) items is more likely to draw participants' attention to the fact that they do not remember the suggested items and, hence, that the descriptions they are being pressed to provide are mere fabrications. To the extent this information is recalled at test, this should reduce misattributions of the suggested items to the eyewitness event (for a discussion of the role of discrepancy detection 
in reducing suggestibility, see Tousignant, Hall, \& Loftus, 1986). Similarly, pressing participants to describe suggested items is more likely to lead to an affective response (e.g., reluctance or discomfort) than is simply reading detailed descriptions of suggested items. This affective response might provide another powerful cue that the suggested information was not, in fact, witnessed. Note that the reluctance and discomfort that a witness presumably feels when pressed to describe a suggested item is less likely to be present in situations in which a person is asked simply to imagine a suggested item or event, because doing so does not commit him or her to endorsing the imagined version as "true." Therefore, the finding that imagining suggested events increases false memory (e.g., Dobson \& Markham, 1993; Hyman \& Pentland, 1996) does not necessarily imply that pressing witnesses to describe suggested events would similarly do so. Thus, our analysis suggests that the effect of generating descriptions on witness suggestibility is dependent on the type of features that are activated during the generation process and subsequently retrieved at the time of test.

We conducted two experiments to assess the impact of generating descriptions of suggested items on eyewitness suggestibility. In both experiments, participants saw a slide sequence of a mock crime and, $48 \mathrm{~h}$ later, read some paragraphs describing the crime that contained misleading postevent suggestions and answered some questions requesting additional details about specific items seen in the event or suggested items that were not present. Five days later, participants completed a source memory test that included judgments of recollective experience. Correct attributions of the suggested items to the postevent paragraphs served as a measure of accurate memory for its actual source, and misattributions of the suggested items to the slides served as the measure of false memory for having witnessed the items. In Experiment 1, we used a within-subjects paradigm to compare the impact of having participants (1) generate two perceptual details describing the appearance of suggested items (generate-elaboration condition), (2) read two perceptual details about the appearance of the suggested items (read-elaboration condition), and (3) read the suggested item alone - that is, with no details about its appearance (read-item-only condition, a standard procedure for eyewitness suggestion in the literature). The questions of primary interest were (1) whether generating descriptions of suggested items would lead participants to misattribute the suggested items to the witnessed event at a higher rate than would reading an elaborated version of the suggested item and (2) whether the recollective experience that accompanied false memories for suggested items would differ in the generate and the read conditions, in such a way that false memories that resulted from generation would more likely be experienced as reliving the episode.

\section{EXPERIMENT 1}

\section{Method}

Participants. Sixty undergraduates participated for course credit in their psychology course.
Materials and Procedure. The experiment took place in three phases. Participants were run in groups of 5-12. In the first phase, participants were told that the experiment concerned people's interpretations of complex events and that their task would be to view a series of slides and attempt to figure out what the incident depicted in the slides was about. Participants viewed a slide sequence that was a modified version of that used by McCloskey and Zaragoza (1985). The series of 79 slides depicts an incident in which a maintenance man enters an office, repairs a chair, finds and steals $\$ 20.00$ and a calculator, and leaves. Slides were shown at a rate of 1 every $4 \mathrm{sec}$.

The second phase took place $48 \mathrm{~h}$ later. Participants were told that they were going to read some paragraphs describing the event that they had seen at the previous session and that, at regular intervals, they would be asked to provide two details about a particular object from the slides that was mentioned in the paragraph. They were instructed to report details such as color, shape, brand name, or where the object was located and were told that the details should be specific to the object that they had seen in the slides. Participants were told to generate the details, even if they had to guess. The postevent paragraphs/questions were presented to participants on a computer and consisted of 12 paragraphs (each paragraph two to three sentences in length) that provided a detailed description of the incident depicted in the slides. For each paragraph, participants read the sentences and then provided two details describing a specific object mentioned in the sentences. Nine of the 12 paragraphs were misleading in that they presupposed the existence of an item that was not in the slide sequence. A total of 12 misleading items were used in the experiment: coffee can, wallet, soda can, paperback book, rag, xerox machine, hammer, lighter, bubblegum, wristwatch, coat rack, and newspaper. Each participant was exposed to a total of 9 of these misleading items, with 3 items assigned to each of the following conditions: generate-elaboration, read-elaboration, and read-itemonly. The remaining 3 items served as never-presented (control) items on the final test. For example, participants for whom the suggested item wristwatch was assigned to the generate-elaboration condition received the following:

The man closed his dark gray, metal toolbox and walked towards the door. He glanced at his wristwatch. The man appeared anxious, but opened the door, stepped outside the office, and closed the door behind him.

Please tell me two things about the wristwatch that was shown in the slides.

Participants for whom the suggested item wristwatch was assigned to the read-elaboration condition received the following:

The man closed his toolbox and walked towards the door. He glanced at a black wristwatch on his left wrist. The man appeared anxious when he looked at his wristwatch, but opened the door, stepped outside the office, and closed the door behind him.

Please tell me two things about the toolbox that was shown in the slides.

Participants for whom the suggested item wristwatch was assigned to the read-item-only condition received the following:

The man closed his toolbox and walked towards the door. He glanced at his wristwatch. The man appeared anxious when he looked at his wristwatch, but opened the door, stepped outside the office, and closed the door behind him.

Please tell me two things about the toolbox that was shown in the slides.

Finally, participants for whom wristwatch was a control (never seen or read) item received the following: 
The man closed his toolbox and walked towards the door. He glanced out the window. The man appeared anxious, but opened the door, stepped outside the office, and closed the door behind him.

Please tell me two things about the toolbox that was shown in the slides.

Across the experiment, each of the 12 suggested items served equally often in each condition. Participants generated details for the suggested item in the generate-elaboration condition only. In the remaining paragraphs, the item for which details were generated (e.g., toolbox) was actually seen in the slide sequence. Thus, the act of generating a description was not unique to suggested items. The details that described suggested items in the read-elaboration condition are shown in Appendix A.

Five days later, participants returned for the third and final phase of the experiment. At this time, they were informed that they were going to receive a 30 -item source test and that, for each item, they were to answer two questions using a 7-point confidence scale: (1) "Did you see the item in the slides?" and (2) "Did you read the item in the paragraphs/questions?" Participants were told that the items on the test were of four basic types: Some had only been seen in the slides, some had only been read in the paragraphs/questions, some had been both seen in the slides and read in the questions, and some were neither seen in the slides nor read in the paragraphs/ questions (see Zaragoza \& Lane, 1994). Furthermore, each time they answered one of the questions with a confidence level of $1,2,3$, or 4 (definitely yes, probably yes, maybe yes, or unsure), they would be asked to answer two additional questions (adapted from Brewer, 1988) about their recollective experience of that item, using a 7-point scale: (1) "While you were recalling the item above, to what extent were you re-experiencing the way the item looked visually in the slides (while reading the item in the paragraphs/questions)?" and (2) "While you were recalling the item above, to what extent were you re-experiencing the context in which you saw the item (in which you read the item)?" The source memory test consisted of a list of 30 items that were presented on computer. Importantly, the specific details that were generated or read by participants during the postevent session were not re-presented at the time of test. The test list for participants was constructed in such a way that, for each participant, there were (1) 3 suggested items from each of three categories (nine total) - suggested items for which details had been generated in the paragraphs/questions, suggested items that had been read in elaborated form in the paragraphs/questions, and suggested items that had been read in the paragraphs/questions in unelaborated form-(2) 3 control items that were not presented in the slides or the postevent questions, (3) 9 true items that had been seen in the slides and for which details had been generated in the paragraphs/ questions and 3 true items that had been seen in the slides and read in elaborated form in the paragraphs/questions, (4) 3 true items that had been seen in the slides but not mentioned in the paragraphs/ questions, and (5) 3 never-presented filler items. Test items from these categories were randomly intermixed throughout the test list, and the same list was presented to all participants. Hence, the category of critical test items was determined by the specific version of the postevent paragraphs the participant had read and the specific objects they were asked to describe.

\section{Results}

In the following analyses, we focus on the attributions of suggested items on the source test. A yes response to the question, "Did you read the item in the paragraphs/ questions?" served as the measure of memory for the suggested items' actual source, and a yes response to the question, "Did you see the item in the slides?" served as the measure of false memory. For each measure, we present analyses of total yes responses (the sum of definitely yes, probably yes, and maybe yes responses) and analyses restricted to highly confident responses (i.e., definitely yes responses). Unless otherwise noted, the results were significant at the .05 alpha level.

Accurate attributions to the paragraphs. We first examined the impact of type of processing (i.e., generate-elaboration, read-elaboration, or read-item-only) on participants' ability to correctly attribute postevent suggestions to the paragraphs. The analysis revealed that type of processing had a substantial impact [Figure 1A; $\left.F(2,118)=87.16, M S_{\mathrm{e}}=0.054, \eta_{\mathrm{p}}^{2}=.60\right]$ on source accuracy. Specifically, generating details about suggested items led to significantly more accurate attributions $(M=$ .65) than did reading an elaborated version of the items $(M=.17)$ or reading the items alone $(M=.16)$. The same pattern was seen in highly confident (definitely read) attributions of suggested items to the questionnaire $[M=.43$, .06 , and $\left..04 ; F(2,118)=55.2, M S_{\mathrm{e}}=0.053, \eta_{\mathrm{p}}^{2}=.48\right]$. In both analyses, performance in the read-elaboration and read-item-only conditions did not significantly differ.

Misattributions to the slides. We next examined the impact of type of processing on participants' erroneous attributions of suggested items to the witnessed event (i.e., false memory). Despite the increased accuracy noted above, type of processing also led to a large increase in misattribution errors [see Figure 1B; $F(2,118)=12.69$, $\left.M S_{\mathrm{e}}=0.07, \eta_{\mathrm{p}}^{2}=.18\right]$. As is obvious from the figure, generating perceptual details $(M=.58)$ led to significantly higher source misattribution errors than did either reading perceptual details about suggested items $(M=$ $.37)$ or reading the suggested items alone $(M=.37)$. The same pattern was also obtained when the analysis was restricted to responses in which participants claimed that they definitely saw the suggested items in the event $[M=$ $.41, .20$, and $.17 ; F(2,118)=18.42, M S_{\mathrm{e}}=0.06, \eta_{\mathrm{p}}^{2}=$ .24]. As with accurate attributions, analyses revealed no significant differences between the read-elaboration and read-item-only conditions. Taken with the previous results, generating descriptions of suggested items increased both accurate memory for having read the suggested items and false memory for having seen the suggested items in the eyewitness event.

Finally, although generating elaborations produced much higher rates of false memory for having witnessed the suggested items than did the two read conditions, it was still the case that exposure to suggested information in the read-elaboration and read-item-only conditions also produced false memory (i.e., a reliable misinformation effect). Specifically, regardless of processing condition, participants were significantly more likely to misattribute the suggested items to the slides if they had been exposed to the suggestion $(M=.58, .37$, and .37 for generate, readelaboration, and read-item-only conditions, respectively) than if they had not $(M=.23$ for the control items, all $p$ s $<.05$; see also Figure 1B).

Recollective experience of false memories. Given that a participant has misattributed a suggested item to the event (definitely, probably, or maybe "saw"), what is the nature of their recollective experience? We examined participants' ratings of their recollective experience on 
A

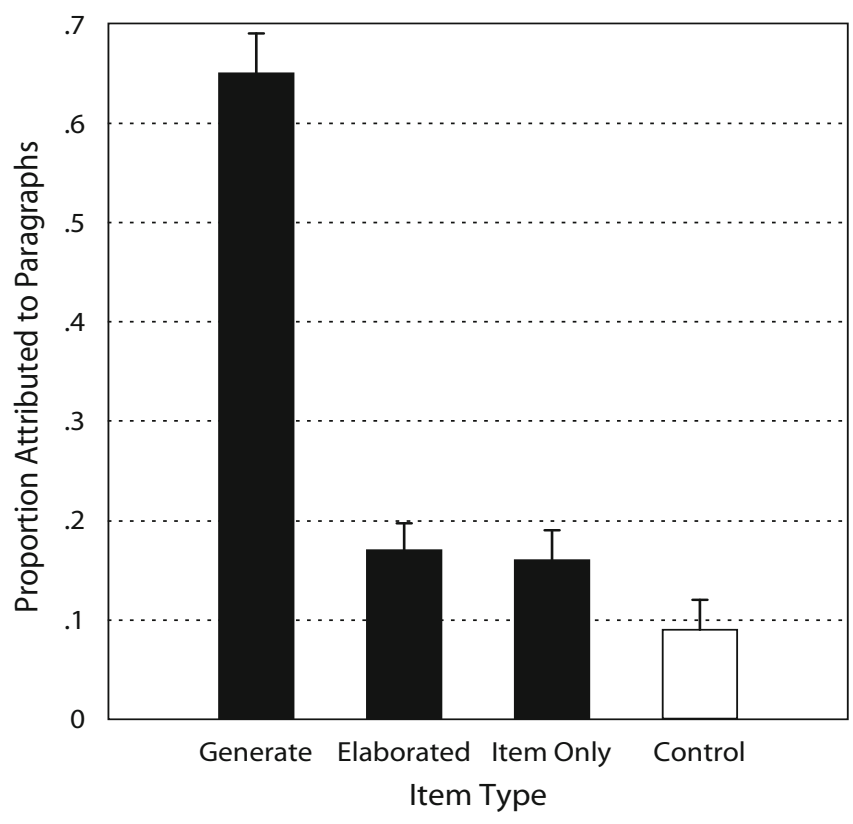

B

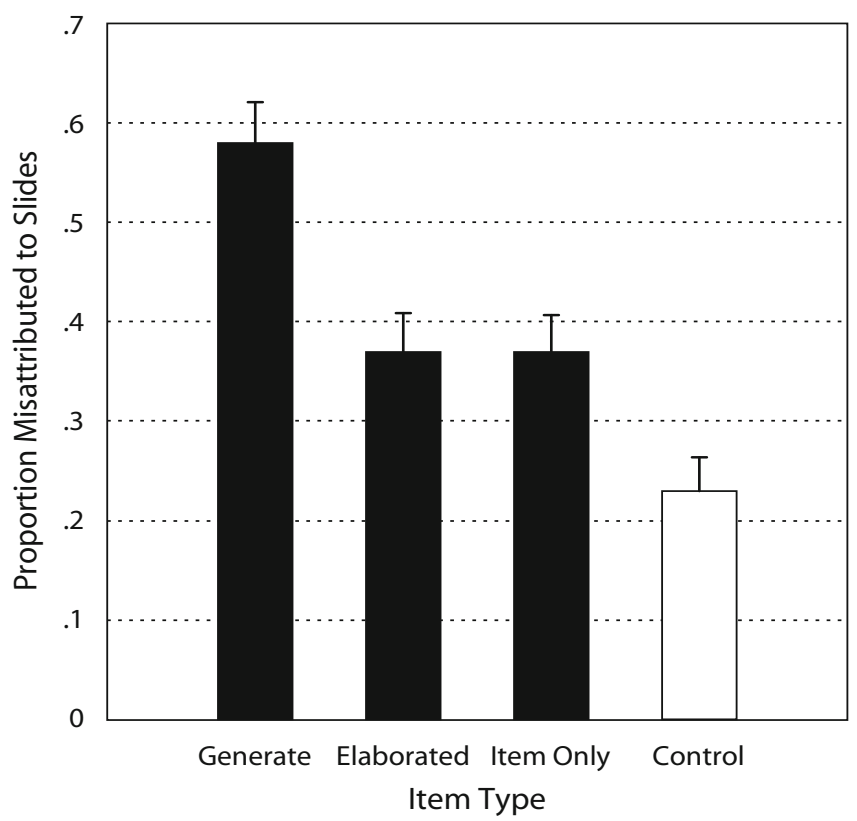

Figure 1. (A) Mean proportion of yes responses to the "Read in the Paragraphs?" test question as a function of type of processing in Experiment 1. Correct attributions of suggested items are illustrated by black bars, and misattributions of control items are illustrated by the white bar for comparison. Error bars represent standard error. (B) Mean proportion of yes responses to the "Saw in the Slides?" test question as a function of type of processing in Experiment 1. Misattributions of suggested items are illustrated by black bars, and misattributions of control items (collapsed across condition) are illustrated by the white bar for comparison.

two dimensions. A comparison of the processing conditions on Qualitative Question 1 ("To what extent were you visually re-experiencing the way the item looked in the slides?") revealed that although the means were in the predicted direction $(M=5.1,4.9$, and 4.7 for generate, read elaboration, and read-item-only conditions, respectively), the overall effect failed to reach significance $[F(2,118)=$ $1.8, M S_{\mathrm{e}}=1.4$, n.s., $\left.\eta_{\mathrm{p}}^{2}=.03\right]$. However, there were significant differences in participants' responses to Qualitative Question 2 ["To what extent were you re-experiencing the context in which you saw the item?"; $F(2,118)=5.6$, $\left.M S_{\mathrm{e}}=1.35, \eta_{\mathrm{p}}^{2}=.09\right]$. Subsequent tests revealed that participants in the generation-elaboration condition $(M=$ 5.4) rated their memory for the context of their false memory significantly higher than did participants in the other two conditions $(M=4.9$ and 4.7 for the read-elaboration and the read-item-only conditions, which did not differ significantly). Hence, it appears that one reason why generating descriptions leads to increased source misattribution errors is that this manipulation leads participants to have an especially vivid false "memory" for the context in which it supposedly appeared.

\section{Discussion}

When descriptions were generated for suggested items, those items were more often misattributed to the witnessed event and more often correctly attributed to the paragraphs than when elaborations of the items or the items alone were read in the context of the postevent paragraphs. Furthermore, generating descriptions led participants to claim that they had a more vivid false memory of the context in which the item was supposedly seen in the event than did reading the other versions of the suggestions. Thus, the evidence is consistent with the interpretation that it is the act of generating perceptual details for a suggested item, and not simply exposure to perceptual detail, that increases false memories of having witnessed suggested items. One potential reason for the increase in false memories is that the generation process led participants to create highly detailed and plausible representations based on their own idiosyncratic knowledge of the event and the item classes (i.e., semantic knowledge).

However, there were several procedural differences between the generate-elaboration and the read-elaboration conditions in Experiment 1 that limit the strength of these conclusions. First, in the generate-elaboration condition, participants did not generate the same details that were read in the corresponding read-elaboration condition. Hence, it is possible that it was the type of descriptions participants generated about the suggested items, and not the act of generation itself, that promoted false memory for the suggestions. A second difference concerned the format of the elaborations. In Experiment 1, participants were prompted to type in the details that they generated but did not type those they read. Rather, in the readelaboration condition, the details were embedded in a postevent narrative that they read (note that this procedure did allow for a clear comparison between elaborated and unelaborated read items). In Experiment 2, we overcame these interpretive difficulties by ensuring that participants in the generate-elaboration and read-elaboration conditions were treated identically, except for the process of generation. 
Finally, the results of Experiment 1 provided no evidence that reading a perceptually detailed description of a suggested item (read-elaboration condition) increased false memory relative to reading about the suggested item alone (read-item-only condition). However, we do not claim on the basis of this one finding that reading such elaborations will never increase false memories. Because the misleading suggestions employed in this study (e.g., rag and hammer) were relatively standard objects that did not lend themselves to a wide variety of descriptors, it is possible that the elaborations participants read were similar to those that were spontaneously activated when participants read the item alone. Hence, whether the present findings would generalize to situations involving other types of suggested items remains an important question for future research. Nevertheless, because we failed to find any effect of reading elaborations in the first experiment, we did not pursue this issue further in Experiment 2. Rather, the purpose of Experiment 2 was to further explore the finding that generating descriptions of suggested items led to higher false memory than did reading descriptions of suggested items.

\section{EXPERIMENT 2}

In Experiment 2, we assessed whether generating perceptual details about a suggested item increased false memory, relative to reading a description of the suggested items containing the same perceptual details. We ensured that the generated details were the same as the read details in two ways. First, the details provided by each participant in a free generation group (who, as in Experiment 1, freely generated two details about each suggested item) were read by a yoked participant in a yoked read group. Although this approach has its strengths, a potential problem would occur if participants in the generation group produced details that seemed unlikely or unusual to participants who only read these details. If so, these details might alert participants in the yoked read group that they had not seen the suggested item in the event.

A second approach that avoids the problem of unlikely descriptors involved constraining the details that participants generated through the use of experimenterprovided fragments (cf. Hoffman et al., 1992). Hence, in Experiment 2, we added a fragment generation group who completed fragments such as b_own (brown) for the suggested item wallet. Participants in the experimenterprovided read (EP read) group read these same details (but in whole-word form) and simply typed them in. Inclusion of the fragment generation group also allowed us to assess the impact of generation when the task reduced reliance on idiosyncratic event and semantic knowledge. In addition, examination of how the generation conditions (free generation and fragment generation) performed relative to their control groups (yoked read and EP read, respectively) was informative with respect to the effects of constrained and unconstrained generation of details on false memory.

The qualitative questions that were used in the first experiment as a measure of recollective experience were replaced by a remember/know judgment (Tulving, 1985; see also Gardiner, 1988). Although there were a number of reasons for doing so, the primary reason was to facilitate comparison with our previous work examining recollective experience of false memories (e.g., Lane \& Zaragoza, 1995; Zaragoza \& Mitchell, 1996). We note that traditional remember/know instructions emphasize memory for both the item and the context in which the item was encoded, so the judgment could be said to encompass both aspects of our qualitative questions used in Experiment 1.

The primary hypotheses were that participants who generate descriptions (free generation and fragment generation groups) for suggested items will more often misattribute the items to the witnessed event and will show increased "remembering" of these errors than will participants who read the same descriptions (yoked read and EP read groups, respectively). A secondary hypothesis was that differences between generate and read conditions would be larger under unconstrained than under constrained generation.

\section{Method}

Participants. Two hundred eighty-eight undergraduate students participated for course credit in their psychology course, with 72 participants randomly assigned to each experimental group (free generation, yoked read, fragment generation, and EP read).

Materials and Procedure. The first phase of the experiment was identical to Experiment 1 . In the second phase ( 2 days later), participants read 12 paragraphs on a computer, which gave a summary of the event in the slides. Six of the paragraphs were misleading in that they presupposed the existence of an item that was not in the slide sequence that participants saw. A total of nine misleading items was used across the experiment: newspaper, wallet, watch (modified from wristwatch), coffee jar (formerly coffee can), pack of bubblegum, hammer, rag, paperback, and copy (formerly xerox) machine. Each participant received a total of six suggested items. The remaining three critical items were never-presented (control) items that appeared only on the final test. Three versions of the paragraphs were used across the experiment, so that each misleading item was suggested in two of the versions and functioned as a control item once. After participants had read each paragraph, they were prompted to press the return key on the keyboard. The target item (six of the target items were suggested items, whereas six were items that had actually been seen in the slides) was then presented on the screen, and participants were prompted to follow the procedure consistent with their condition. In the free generation group, participants generated two details for each target item. In the yoked read group, participants read two details (generated by a yoked participant in the free generation group) for each target item and typed them in. In the generate fragment group, participants generated two details from experimenter-provided word fragments (e.g., b_own for the suggested item wallet) for each target item. When a word fragment was not completed correctly, the whole word (detail) was presented to the participant for $2 \mathrm{sec}$. In the EP read group, participants read two (experimenter-provided) details for each target item that were the same details as those used in the generate fragment group and typed these details in. Participants in all the conditions had as much time as they needed to generate and type in (or read and type in) each detail. The time from target item presentation until the second detail was entered was recorded for each item.

For participants in the generate fragment and the EP read conditions, half received fragments or details that were unique, and half received fragments or details that were common (see top of Appendix B). The details were chosen using data from a pilot study $(N=102)$ in which participants viewed the slide sequence, read the paragraphs, and were told to generate details for suggested items. 
Participants were informed that the items had not been in the event in the slides but that they were to generate details that likely would describe the objects if they had been present. The details generated by these participants were used to identify unique (reported by no more than 2 participants) and very common (most common details reported for each item) but highly plausible details. We note that the manipulation of type of detail in the fragment generation and EP read conditions had no significant impact on any dependent measure and, therefore, will not be discussed further. Fragments used in the fragment generation condition were constructed with the goal that at least $80 \%$ of participants would correctly complete a particular fragment. A second pilot study using 80 participants was used to empirically determine how often the fragments would be correctly completed. All but two of the fragments were completed at least $80 \%$ of the time. The two fragments that did not reach this criterion were modified to make them easier to complete. The fragments are shown in the bottom of Appendix B.

In the third phase ( 5 days later), participants received a test of memory for source. All the instructions were identical to those in Experiment 1, except that participants were instructed to make remember/know judgments (Tulving, 1985) each time they answered yes (definitely, probably, or maybe) to a question about the source of the items. The specific instructions participants were given about the remember/know judgments were a modified version of those used by Lane and Zaragoza (1995). Participants were given the option of indicating that they were unsure about their recollective experience, rather than being forced to choose between the remember and the know options. The source memory test consisted of a list of 25 items presented on a computer. The test list for participants was constructed so that for each participant, there were (1) 6 suggested items, (2) 3 never-presented (control) items, (3) 13 true test items that had been seen in the slides and read in the paragraphs/questions, (4) 2 true test items that had been seen in the slides but not mentioned in the paragraphs/questions, and (5) 1 never-presented filler item. Test items from these categories were randomly intermixed throughout the test list, and the same list was presented to all participants Whether a particular critical item was a suggested item or a control item depended on the narrative (paragraphs) version that they had received in the second phase of the experiment.

\section{Results and Discussion}

We began by analyzing participants' misattributions of control items to the slides. A one-way ANOVA revealed no significant differences between the groups $[F(1,284)=$ $1.47]$, with an overall mean of $24 \%$. Consequently, all of the analyses reported below were conducted on participants' test responses to the suggested items only. We also note that there was a significant misinformation effect in each of the four groups. This was evidenced by the fact that, in each group, participants were more likely to misattribute the suggested items to the slides than they were the never-presented control items (all $p \mathrm{~s}<.05$; see also Figure 2B).

In the following sections, we will focus both on the results of omnibus analyses and on specific comparisons between groups that generated and read the same details. In these analyses, we first conducted a $2 \times 2$ factorial ANOVA with type of processing (generate vs. read) and source of detail (participant vs. experimenter provided). ${ }^{3}$
A

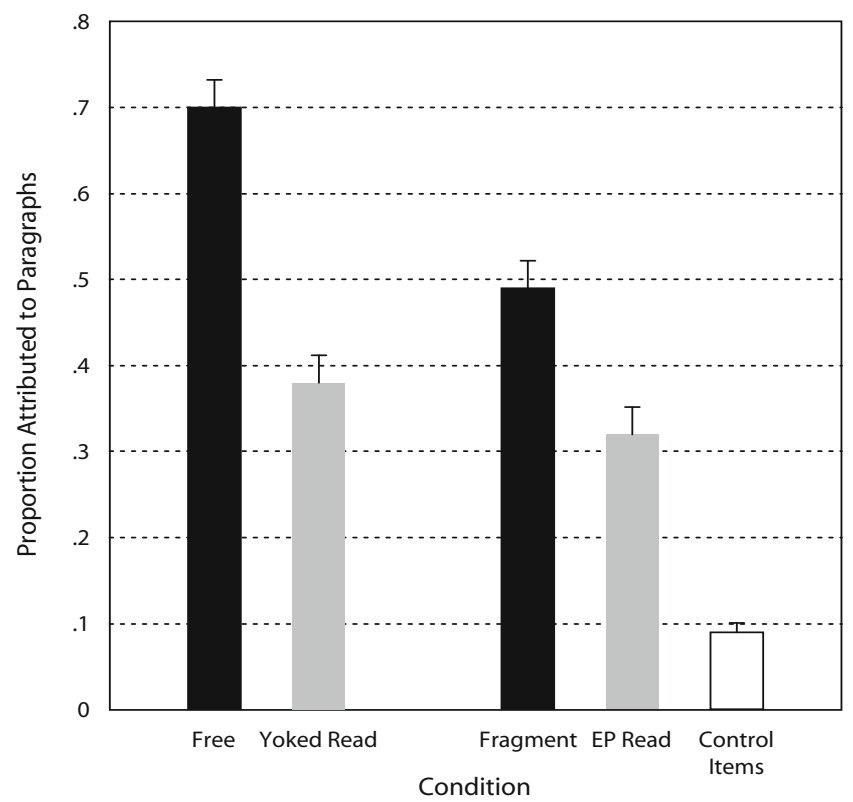

B

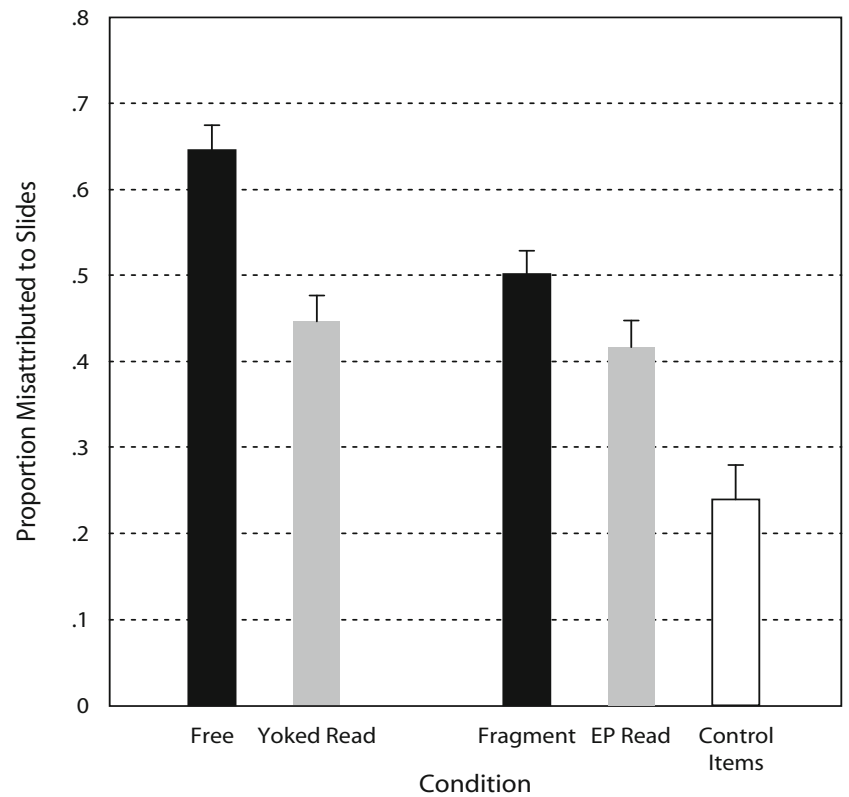

Figure 2. (A) Mean proportion of yes responses to the "Read in the Paragraphs?" test question as a function of generation (generate vs. read) and detail source (subject generated vs. experimenter generated) in Experiment 2. Free and fragment generation conditions involved unconstrained and constrained generation, respectively. Correct attributions of suggested items are illustrated by filled bars, and misattributions of control items (collapsed across condition) are illustrated by the white bar for comparison. Pure black bars represent the generation conditions, and gray bars indicate the read conditions. Error bars represent standard error. EP, experimenter provided. (B) Mean proportion of yes responses to the "Saw in the Slides?" test question as a function of type of processing (generate vs. read) and detail source (participant provided vs. experimenter provided) in Experiment 2. Misattributions of suggested items are illustrated by filled bars, and misattributions of control items (collapsed across condition) are illustrated by the white bar for comparison. Pure black bars represent the generation conditions, and gray bars indicate the read conditions. 
Accurate attributions to paragraphs. Figure 2A portrays overall accurate attributions (collapsed across confidence level) of suggested items to the paragraphs as a function of condition. A $2 \times 2$ factorial ANOVA revealed significant main effects for type of processing $[M=.60$ and .35 for generate and read conditions, respectively; $\left.F(1,284)=60.3, M S_{\mathrm{e}}=0.07, \eta_{\mathrm{p}}^{2}=.18\right]$ and source of detail $[M=.54$ and .41 for participant- and experimenterprovided detail; $\left.F(1,284)=8.7, M S_{\mathrm{e}}=0.07, \eta_{\mathrm{p}}^{2}=.06\right]$. There was also a significant interaction $[F(1,284)=5.7$, $\left.M S_{\mathrm{e}}=0.07, \eta_{\mathrm{p}}^{2}=.02\right]$. Simple effects analyses revealed significantly higher accurate attributions in the free generation condition $(M=.70)$ than in the fragment generation condition $(M=.49)\left[F(1,142)=24.2, M S_{\mathrm{e}}=0.07\right.$, $\left.\eta_{\mathrm{p}}^{2}=.15\right]$ but no significant difference between the two read conditions $(M=.38$ and .32 for yoked read and EP read, respectively; $F<1.5$ ). Each generation condition significantly increased accurate attributions, relative to its content-equated control group $\left[F(1,142)=50.6, M S_{\mathrm{e}}=\right.$ $0.08, \eta_{\mathrm{p}}^{2}=.26$, for free generation and yoked read comparison; $F(1,142)=14.7, M S_{\mathrm{e}}=0.07, \eta_{\mathrm{p}}^{2}=.09$, for fragment generation and EP read comparison]. When the same analyses were restricted to correct attributions in which participants were certain (definitely in the paragraphs), the pattern was exactly the same $(M=.55, .32, .19$, and .19 for free generation, fragment generation, yoked read, and EP read, respectively).

Misattributions to the slides. We next analyzed participants' misattributions of suggested items to the slides, using the same $2 \times 2$ factorial ANOVA described above. Figure $2 \mathrm{~B}$ portrays overall misattributions (collapsed across confidence level) of suggested items to the slides as a function of condition. The analysis revealed significant main effects for type of processing $[M=.57$ and .43 for generate and read conditions, respectively; $F(1,284)=$ $\left.23.5, M S_{\mathrm{e}}=0.06, \eta_{\mathrm{p}}^{2}=.08\right]$ and source of detail $[M=.55$ and .46 for participant- and experimenter-provided detail; $\left.F(1,284)=8.7, M S_{\mathrm{e}}=0.06, \eta_{\mathrm{p}}^{2}=.03\right]$, but these main effects were qualified by an interaction $[F(1,284)=3.7$, $\left.M S_{\mathrm{e}}=0.06, p<.055, \eta_{\mathrm{p}}^{2}=.01\right]$. Simple effects analyses revealed significantly higher misattributions in the free generation condition $(M=.65)$ than in the fragment generation condition $\left[M=.50 ; F(1,142)=3.4, M S_{\mathrm{e}}=\right.$ $\left.0.06, \eta_{\mathrm{p}}^{2}=.08\right]$ but no significant difference between the two read conditions $(M=.45$ and .42 for yoked read and EP read, respectively; $F<1$ ). As would be expected from the foregoing analyses, each generation condition significantly increased source misattribution errors, relative to its content-equated read group $[F(1,142)=22.4$, $M S_{\mathrm{e}}=0.06, \eta_{\mathrm{p}}^{2}=.14$, for free generation and yoked read comparison; $F(1,142)=4.4, M S_{\mathrm{e}}=0.06, \eta_{\mathrm{p}}^{2}=.03$, for fragment generation and EP read comparison]. When the same analyses were restricted to misattributions in which participants were certain (definitely in the slides), the pattern was even stronger. Again, there were significant main effects for type of processing $[M=.39$ and .24 for generate and read conditions, respectively; $F(1,284)=$ $\left.32.8, M S_{\mathrm{e}}=0.05, \eta_{\mathrm{p}}^{2}=.10\right]$ and source of detail $[M=$ .35 and .28 for participant- and experimenter-provided detail; $\left.F(1,284)=8.0, M S_{\mathrm{e}}=0.05, \eta_{\mathrm{p}}^{2}=.03\right]$ and a sig- nificant interaction $\left[F(1,284)=7.6, M S_{\mathrm{e}}=0.06, \eta_{\mathrm{p}}^{2}=\right.$ .03]. Simple effects analyses revealed significantly higher misattributions in the free generation condition $(M=$ $.47)$ than in the fragment generation condition $(M=.32)$ $\left[F(1,142)=14.6, M S_{\mathrm{e}}=0.06, \eta_{\mathrm{p}}^{2}=.08\right]$ but no significant difference between the two read conditions $(M=.24$ and .24 for yoked read and EP read, respectively; $F<1$ ). Each generation condition significantly increased source misattribution errors, relative to its content-equated read group $\left[F(1,142)=35.7, M S_{\mathrm{e}}=0.05, \eta_{\mathrm{p}}^{2}=.20\right.$, for free generation and yoked read comparison; $F(1,142)=4.4$, $M S_{\mathrm{e}}=0.05, \eta_{\mathrm{p}}^{2}=.03$, for fragment generation and EP read comparison].

The analyses above present a clear picture. First, as in Experiment 1, generating perceptual details for suggested items increased source misattributions to the slides and accurate attributions to the paragraphs, relative to reading those same details. This is seen both in significant main effects of generation and in direct comparisons between content-equated generation and read conditions. Second, we were able to rule out the potential concern that yoked read condition participants might find the details (elaborations) generated by free generation participants to be strange or unusual and retrieve this information to reject the suggested items at test. The read conditions did not differ significantly on any measure. Third, although both types of generation increased misattributions and accurate attributions of suggested items, the findings reported above show that freely generating perceptual details had a stronger impact on both measures than did generating details from fragment cues. Because the fragment generation task can be completed by relying on general linguistic or semantic knowledge, these findings suggest that there is a greater impact when idiosyncratic event or semantic knowledge is used in the generation process. Despite a somewhat weaker impact, we note that even generating details from experimenter-provided fragments clearly increases false memories for suggestions, relative to exposure to those details alone.

Recollective experience of false memories. As in Experiment 1, we were interested in the nature of the recollective experience that accompanied false memories of seeing suggested items in the event. The proportion of times participants remembered seeing a misleading item in the slides (rather than simply knowing it had been there), given that they committed a source misattribution error (definitely, probably, or maybe "saw"), was analyzed. A $2 \times 2$ factorial ANOVA revealed significantly greater $r e-$ membering of source misattributions in the generate conditions $(M=.49)$ than in the read conditions $(M=.40)$ $\left[F(1,284)=4.6, M S_{\mathrm{e}}=0.13, \eta_{\mathrm{p}}^{2}=.02\right]$, no significant effect of source of detail $(F<1)$, and an interaction that was shy of statistical significance $\left[F(1,284)=4.6, M S_{\mathrm{e}}=\right.$ $\left.0.13, p=.07, \eta_{\mathrm{p}}^{2}=.01\right]$. Given the hypotheses outlined in the introduction, we decided to examine this finding. Simple effects analyses revealed that the misattributions of free generation participants were significantly more likely to be remembered $(M=.55)$ than were the misattributions of yoked read participants $(M=.38)[F(1,142)=$ $\left.8.1, M S_{\mathrm{e}}=0.13, \eta_{\mathrm{p}}^{2}=.05\right]$. In contrast, the misattribu- 
tions of fragment generation participants were not more often remembered $(M=.44)$ than were the misattributions of EP read participants $(M=.42)(F<1)$. Finally, the source misattributions of free generation $(M=.55)$ participants were more likely to be remembered than were those of fragment generation participants $(M=.44)$ $\left[F(1,142)=3.8, M S_{\mathrm{e}}=0.13, p=.053, \eta_{\mathrm{p}}^{2}=.03 ; p<.05\right.$ with one-tailed comparison], and there was no significant difference between the two read conditions $(F<1)$. As in Experiment 1, participants who generated descriptions freely and later committed source misattribution errors appeared to have an especially vivid conscious recollection of having seen the suggested items in the slides.

Time to produce responses. We also examined the length of time it took participants to type in their responses as a proxy for processing time. A $2 \times 2$ factorial ANOVA revealed a significant main effect of type of processing $[M=24.2$ and $14.3 \mathrm{sec}$ for generate and read, respectively; $F(1,284)=142.2, M S_{\mathrm{e}}=49.1, \eta_{\mathrm{p}}^{2}=.33$, source of detail $[M=21.8$ and $16.7 \mathrm{sec}$ for participant and experimenter provided; $F(1,284)=36.9, M S_{\mathrm{e}}=49.1$, $\left.\eta_{\mathrm{p}}^{2}=.12\right]$, and a significant interaction $[F(1,284)=17.1$, $\left.M S_{\mathrm{e}}=49.1, \eta_{\mathrm{p}}^{2}=.06\right]$. Simple effects analyses revealed that generating perceptual details freely took longer than did generating details from fragment cues $[M=28.4$ and $\left.20 \mathrm{sec} ; F(1,142)=43.3, M S_{\mathrm{e}}=59.0, \eta_{\mathrm{p}}^{2}=.23\right]$, but there was no significant difference between the read conditions $[M=15.1$ and $13.5 \mathrm{sec}$ for yoked read and EP read; $F(1,142)=2.4, M S_{\mathrm{e}}=39.2$, n.s., $\left.\eta_{\mathrm{p}}^{2}=.02\right]$. These differences raise the possibility that the impact of generation is simply a function of greater time spent processing the suggested item. We assessed this possibility two ways. First, we conducted ANCOVAs on the previously reported dependent measures of interest (source misattributions and source accuracy), with time to generate as a covariate, and replicated the pattern of results reported above [e.g., misattributions of suggestions to the slides, free generation vs. yoked read, $\left.F(1,141)=16.6, M S_{\mathrm{e}}=0.06, \eta_{\mathrm{p}}^{2}=.11\right]$. Second, if processing time is the important underlying element, we might expect that processing time should be correlated with source accuracy and source error within a given group. Toward this end, we examined correlations between time to generate and the dependent measures for each group. These analyses revealed no significant associations and no consistent patterns (e.g., in the free generation condition, $r \mathrm{~s}$ ranged from .15 to -.15 , all $p \mathrm{~s}>$ .20). These analyses thus suggest that it is the type, rather than the amount, of processing that was the locus of the generation effects observed in this experiment.

\section{GENERAL DISCUSSION}

The results of two experiments present a consistent picture of the impact of generating descriptions of postevent suggestions on memory for those items. First, generating descriptions, relative to reading them, led to a dramatic increase in the misattribution of suggested items to the witnessed event. Furthermore, these false memories were confidently held and were accompanied by the experience of remembering more often than were the false memories that resulted from reading the descriptions (or reading the suggested item alone). This difference in recollective experience between generate and read conditions was observed even though the suggested items themselves were not generated (e.g., Ackil \& Zaragoza, 1998; Zaragoza et al., 2001); rather, participants generated (or read) details describing the suggested item that appeared on the test. Note also that these descriptions affected false memory for the suggested items even though the details were not re-presented at the time of test. Collectively, the results show that it is the act of generating perceptual details, and not simply exposure to them, that is the key factor in increasing false memories.

Second, almost paradoxically, generation led to an increase in false memory even though it also improved participants' memory for having encountered the suggested item in the postevent paragraphs. Consistent with previous research on the effect of generation on source memory (e.g., Dewhurst \& Hitch, 1999; Marsh \& Hicks, 1998), it is likely that, relative to reading descriptions, generating descriptions of suggested items increased memory for the cognitive operations associated with the generation process, thus improving participants' memory that the suggestion was encountered in the postevent source. However, the knowledge that the suggested item was from the postevent source did not allow participants to ascertain whether or not the item was also witnessed in the original event, because most of the information encountered in the postevent narrative was true. Indeed, participants had also generated descriptions of items that they had actually witnessed. To be able to reduce false memories, participants who generated descriptions would need to have access to more specific information (thoughts, affective reactions) that indicated that they had fabricated the description of the suggested item. Although participants in our study often seemed to be aware that they could not remember the items that they were being asked to describe (e.g., from verbal comments made during the suggestion phase), they nevertheless failed to remember this information at the time of test. Thus, in this study, there was no evidence that generation reduced suggestibility by increasing discrepancy detection (Tousignant et al., 1986).

Finally, the fact that generation was more potent when it was unconstrained than constrained (through the use of fragment cues) suggests that an important factor in the increased error rate is the degree to which people must invent the details on their own. It is possible that having to invent details of your own choosing involves a greater investment and commitment to the response than does producing the details from experimenter-provided cues (see Schooler, Foster, \& Loftus, 1988; Schreiber \& Sergent, 1998, for evidence that commitment to misinformation has consequences for memory). In addition, having to generate descriptions of suggested items may result in elaborations that are more plausible than those provided by someone else.

The results of both experiments serve to clarify the hypothesized mechanisms outlined in the introduction. Generating descriptions of suggested items substantially increased misattributions to the slides and increased the 
proportion of time that these errors were accompanied by a sense of being reexperienced or remembered as having been in the event. We propose that a major factor underlying the impact of generating perceptual details on suggestibility concerns the reflective processes that are elicited by this manipulation. We first note that these processes may sometimes be elicited when participants are exposed to suggestions in the context of elaborated descriptions or the standard methods used in the eyewitness suggestibility literature, but not as consistently or broadly and not to the same degree. When witnesses are pressed to describe the physical appearance of a suggested item that they do not remember, they are likely to construct a mental model (e.g., Zwaan \& Radvansky, 1998) based on their memory for the event and the knowledge they possess about the characteristics of the item class. As such, participants may be more likely to construct and encode a richer, more elaborate representation of what the item looked like [e.g., in the case of the suggested item hammer, details such as wooden handle, claw, or (had a) black head] than when the item is read in a narrative or presupposed in a question or when perceptual details are simply described (for similar arguments with respect to the effect of type of postsuggestion review, see Lane, Mather, Villa, $\&$ Morita, 2001). Furthermore, given the obtained differences between unconstrained and constrained generation in both the incidence of false memories and the recollective experience that accompanied those memories, it is likely that utilizing idiosyncratic event or semantic knowledge during generation may later make the false memories of suggested items particularly plausible. To the extent that a memory representation contains features that "feel like" something he or she would have noticed or reflected about during the event, such features are likely to be compelling evidence for having witnessed it (see also Stark et al., 2005; Zaragoza et al., 2001). In the context of the source-monitoring framework (e.g., Johnson et al., 1993), false memories created by generated descriptions are thus likely to be misattributed to the witnessed event because they contain characteristics that would be expected of event items.

Like many aspects of human cognition, the ability to speculate is responsible for both impressive feats and consequential errors. For example, the ability to mentally simulate future activity plays an important role in behavioral and emotional regulation and, ultimately, health (for a review, see Taylor, Pham, Rivkin, \& Armor, 1998). Furthermore, this ability is central to scientists' ability to make hypotheses or conjectures, and there is evidence that we use constructed mental models to reason about situations with which we have had no direct experience (e.g., Johnson-Laird, 1983) or when trying to comprehend language (for a review, see Zwaan \& Radvansky, 1998). On the other hand, when a premium is placed on accurately remembering the source of information (e.g., when testifying in a court case), this ability may not always serve us well. Our results suggest that pressing witnesses to describe the physical appearance of suggested items (e.g., objects, actions, or people) that they do not remember witnessing is likely to have unintended consequences. Even when such descriptions are not re-presented to witnesses, the negative effects persist, in such a way that they are more likely to falsely remember witnessing the suggested items and more likely to recollect them vividly.

\section{AUTHOR NOTE}

The authors thank Glenn Clayton, Laurie Crawford, and Laura Holland for their help collecting the data, Aimee Benson for assistance scoring the qualitative data, Paul Atchley for writing the computer program used in this study, and Ken Chambers for helping make several important programming changes. The authors also thank Yossef Ben-Porath, Joseph Danks, William Merriman, and Jason Hicks for their comments on an earlier version of the manuscript. Correspondence regarding this article should be addressed to S. M. Lane, Office of Applied Cognition, Department of Psychology, Louisiana State University, 236 Audubon Hall, Baton Rouge, LA 70803 (e-mail: slane@1su.edu).

\section{REFERENCES}

ACKIL, J. K., \& ZaragozA, M. S. (1998). Memorial consequences of forced confabulation: Age differences in the susceptibility to false memories. Developmental Psychology, 34, 1358-1372.

BREWER, W. F. (1988). Memory for randomly sampled autobiographical events. In U. Neisser \& E. Winograd (Eds.), Remembering reconsidered: Ecological and traditional approaches to the study of memory (pp. 21-90). Cambridge: Cambridge University Press.

BuRNS, D. J. (1990). The generation effect: A test between single- and multifactor theories. Journal of Experimental Psychology: Learning, Memory, \& Cognition, 16, 1060-1067.

DENT, H. R. (1982). The effects of interviewing strategies on the results of interviews with child witnesses. In A. Trankell (Ed.), Reconstructing the past: The role of psychologists in criminal trials (pp. 279-297). Deventer, The Netherlands: Kluwer.

Dewhurst, S. A., \& Hitch, G. J. (1999). Cognitive effort and recollective experience in recognition memory. Memory, 7, 129-146.

DoBSON, M., \& MARKHAM, R. (1993). Imagery ability and source monitoring: Implications for eyewitness memory. British Journal of Psychology, 32, 111-118.

Drivdahl, S. B., \& Zaragoza, M. S. (2001). The role of perceptual elaboration and individual differences in the creation of false memories for suggested events. Applied Cognitive Psychology, 15, 265-281.

GaLlo, D. A. (2004). Using recall to reduce false recognition: Diagnostic and disqualifying monitoring. Journal of Experimental Psychology: Learning, Memory, \& Cognition, 30, 120-128.

GARDINER, J. M. (1988). Functional aspects of recollective experience. Memory \& Cognition, 16, 309-313.

Garry, M., Manning, C. G., Loftus, E. F., \& Sherman, S. J. (1996). Imagination inflation: Imagining a childhood event inflates confidence that it occurred. Psychonomic Bulletin \& Review, 3, 208-214.

Geghman, K. D., \& Multhaup, K. S. (2004). How generation affects source memory. Memory \& Cognition, 32, 819-823.

GofF, L. M., \& Roediger, H. L., III (1998). Imagination inflation for action events: Repeated imaginings lead to illusory recollections. Memory \& Cognition, 26, 20-33.

Gonsalves, B., Reber, P. J., Gitelman, D. R., Parrish, T. B., Mesulam, M.-M., \& Paller, K. A. (2004). Neural evidence that vivid imagining can lead to false remembering. Psychological Science, 15, 655-660.

Hicks, J. L., MARSh, R. L., \& Ritschel, L. (2002). The role of recollection and partial information in source monitoring. Journal of Experimental Psychology: Learning, Memory, \& Cognition, 28, 503-508.

Hirshman, E., \& BJork, R. A. (1988). The generation effect: Support for a two-factor theory. Journal of Experimental Psychology: Learning, Memory, \& Cognition, 14, 484-494.

Hoffman, H. G., Loftus, E. F., Greenmun, C. N., \& Dashiell, R. L. (1992). The generation of misinformation. In F. Lösel, D. Bender, \& T. Bliesener (Eds.), Psychology and law: International perspectives (pp. 292-301). Berlin: de Gruyter.

Hyman, I. E., \& Pentland, J. (1996). The role of mental imagery in the creation of false childhood memories. Journal of Memory \& Language, 35, 101-117. 
Johnson, M. K., Hashtroudi, S., \& Lindsay, D. S. (1993). Source monitoring. Psychological Bulletin, 114, 3-28.

Johnson, M. K., \& RaYe, C. L. (1981). Reality monitoring. Psychological Review, 88, 67-85.

JoHNSON-LAIRD, P. N. (1983). Mental models: Towards a cognitive science of language, inference and consciousness. Cambridge: Cambridge University Press.

Lane, S. M., Mather, M., Villa, D., \& Morita, S. K. (2001). How events are reviewed matters: Effects of varied focus on eyewitness suggestibility. Memory \& Cognition, 29, 940-947.

Lane, S. M., \& Zaragoza, M. S. (1995). The recollective experience of cross-modality confusion errors. Memory \& Cognition, 23, 607-610.

Lindsay, D. S., Hagen, L., Read, J. D., Wade, K. A., \& Garry, M. (2004). True photographs and false memories. Psychological Science, 15, 149-154.

MARSH, R. L., \& Hicks, J. L. (1998). Test formats change sourcemonitoring decision processes. Journal of Experimental Psychology: Learning, Memory, \& Cognition, 24, 1137-1151.

McCloskey, M., \& Zaragoza, M. (1985). Misleading postevent information and memory for events: Arguments and evidence against memory impairment hypotheses. Journal of Experimental Psychology: General, 114, 3-18.

McDaniel, M. A., Wadill, P. J., \& Einstein, G. O. (1988). A contextual account of the generation effect: A three-factor theory. Journal of Memory \& Language, 27, 521-536.

Mitchell, K. J., \& Zaragoza, M. S. (2001). Contextual overlap and eyewitness suggestibility. Memory \& Cognition, 29, 616-626.

Mulligan, N. W. (2004). Generation and memory for contextual detail. Journal of Experimental Psychology: Learning, Memory, \& Cognition, 30, 838-855.

Pickel, K. L. (2004). When a lie becomes the truth: The effects of self-generated misinformation on eyewitness memory. Memory, 12, 14-26.

Schooler, J. W., Foster, R. A., \& Loftus, E. F. (1988). Some deleterious consequences of the act of recollection. Memory \& Cognition, 16, 243-251.

Schreiber, T. A., \& Sergent, S. D. (1998). The role of commitment in producing misinformation effects in eyewitness memory. Psychonomic Bulletin \& Review, 5, 443-448.

Slamecka, N. J., \& Graf, P. (1978). The generation effect: Delineation of a phenomenon. Journal of Experimental Psychology: Human Learning \& Memory, 4, 592-604.

Stark, L. J., Perfect, T. J., \& Newstead, S. (2005). When elaboration leads to appropriation: Unconscious plagiarism in a creative task. Memory, 13, 561-573.

Taylor, S. E., Pham, L. B., Rivkin, I. D., \& Armor, D. A. (1998).
Harnessing the imagination: Mental simulation, self-regulation, and coping. American Psychologist, 53, 429-439.

Thomas, A. Y., Bulevich, J. B., \& Loftus, E. F. (2003). Exploring the role of repetition and sensory elaboration in the imagination inflation effect. Memory \& Cognition, 31, 630-640.

Tousignant, J. P., Hall, D., \& Loftus, E. F. (1986). Discrepancy detection and vulnerability to misleading postevent information. Memory \& Cognition, 14, 329-338.

Tulving, E. (1985). Memory and consciousness. Canadian Psychology, 26, 1-12.

Zaragoza, M. S., \& LANE, S. M. (1994). Source misattributions and the suggestibility of eyewitness memory. Journal of Experimental Psychology: Learning, Memory, \& Cognition, 20, 934-945.

Zaragoza, M. S., \& Mitchell, K. M. (1996). Repeated suggestion and the creation of false memories. Psychological Science, 7, 294-300.

Zaragoza, M. S., Payment, K. E., Ackil, J. K., Drivdahl, S. B., \& BECK, M. (2001). Interviewing witnesses: Forced confabulation and confirmatory feedback increase false memories. Psychological Science, 12, 473-477.

ZwaAn, R. A., \& Radvansky, G. A. (1998). Situation models in language comprehension and memory. Psychological Bulletin, 123, 162185 .

\section{NOTES}

1. There is a literature on the impact of being forced to generate misleading items (forced confabulation; see, e.g., Ackil \& Zaragoza, 1998; Pickel, 2004; Zaragoza et al., 2001). There are, of course, issues that overlap between the two types of situations. However, studies in this literature do not typically compare generating versus reading suggested items. In a study in which this has been done (Pickel, 2004, Experiment 2), participants knew that the suggested items were incorrect at the time that they were provided because they were told to talk about them convincingly in a later interview.

2. We note that Mulligan (2004) has recently shown that generating a studied item sometimes impairs memory for contextual features of the item. So far, this work has focused on memory for incidental aspects of context (color, location) for studied words. The circumstances under which generation may enhance, detract, or have no effect on context memory remains an important question for future research.

3. Performance in the fragment generation condition was also examined contingent on whether participants were successful in generating both details. An analysis of this data revealed that performance did not differ regardless of whether the data was conditionalized on correct completion. Therefore, the data reported in the results are not conditionalized.

\section{APPENDIXA \\ Item Descriptions for the Read-Elaboration Condition in Experiment 1}

\begin{tabular}{ll}
\hline Suggested Items & \multicolumn{1}{c}{ Descriptions } \\
\hline Coat rack & tall, near the desk \\
Coffee can & medium-sized, Maxwell House \\
Rag & dirty, brown \\
Lighter & silver, BIC \\
Newspaper & yellowed, folded in half \\
Paperback & green, with black lettering \\
Wallet & old, brown \\
Xerox machine & gray, with white top \\
Pack of bubblegum & pink, Hubba Bubba \\
Soda can & opened, Pepsi \\
Hammer & silver-colored, with wooden handle \\
Wristwatch & black, on left wrist \\
\hline
\end{tabular}


APPENDIX B

Unique and Common Details and Fragments Used for Suggested Items in the Fragment Generation and EP Read Conditions

\begin{tabular}{|c|c|c|}
\hline Item & Unique & Cor \\
\hline $\begin{array}{l}\text { P Read Condition } \\
\text { Paperback } \\
\text { Wallet } \\
\text { Copy machine } \\
\text { Pack of bubblegum } \\
\text { Hammer } \\
\text { Newspaper } \\
\text { Watch } \\
\text { Rag } \\
\text { Jar of coffee }\end{array}$ & $\begin{array}{l}\text { dog-eared, mystery } \\
\text { overstuffed, snakeskin } \\
\text { unplugged, Toshiba } \\
\text { crumpled, Bazooka } \\
\text { Craftsman, cracked handle } \\
\text { Washington Post, bold print } \\
\text { gold-rimmed, pocket } \\
\text { clean, checkered } \\
\text { opened, yellow scoop (in it) }\end{array}$ & $\begin{array}{l}\text { thick, romance } \\
\text { brown, leather } \\
\text { large, white } \\
\text { open, pink } \\
\text { heavy, wooden handle } \\
\text { folded, daily } \\
\text { digital, black band } \\
\text { dirty, square } \\
\text { round, glass }\end{array}$ \\
\hline $\begin{array}{l}\text { ragment Generation Condition } \\
\text { Paperback } \\
\text { Wallet } \\
\text { Copy machine } \\
\text { Pack of bubblegum } \\
\text { Hammer } \\
\text { Newspaper } \\
\text { Watch } \\
\text { Rag } \\
\text { Jar of coffee }\end{array}$ & $\begin{array}{l}\text { d_g-e_red, m_ste_y } \\
\text { ove_st_ff_d, sn_kesk_n } \\
\text { u_plu_ged, T_sh_ba } \\
\text { cr_mpl_d, B_zo_ka } \\
\text { C_afts_an, cr_ck_d handle } \\
\text { W_shi_gt_n Post, b_ld print } \\
\text { g_ld-r_m_ed, po_k_t } \\
\text { c_ean,c_ecker_d } \\
\text { o_en_d, y_llo_scoop }\end{array}$ & $\begin{array}{l}\text { t_ick, rom_nc_- } \\
\text { b_own, lea_h_r } \\
\text { la_ge, w_ite } \\
\text { op_n,p_nk } \\
\text { hea_y, w_ode_handle } \\
\text { fo_de_,da_ly } \\
\text { d_gita_,_lack band } \\
\text { di_ty, s_uare } \\
\text { rou_d,g_ass }\end{array}$ \\
\hline
\end{tabular}

(Manuscript received August 4, 2006;

revision accepted for publication September 13, 2006.) 